\title{
Índice Apo B/Apo A-I e Predição de Risco Cardiovascular
}

\author{
Apo B/Apo A-I Ratio and Cardiovascular Risk Prediction
}

\author{
Luciana Moreira Lima, Maria das Graças Carvalho, Marinez Oliveira Sousa \\ Universidade Federal de Minas Gerais - Belo Horizonte, MG - Brasil
}

\section{Apolipoproteínas A-I e B}

As apolipoproteínas são proteínas que estão em associação com lípides nas partículas de lipoproteínas e desempenham funções importantes no metabolismo lipoprotéico, como transporte dessas moléculas hidrofóbicas no meio aquoso plasmático, ligação aos receptores específicos na superfície celular para direcionar corretamente os lípides para os órgãosalvo e tecidos do organismo, e ativação ou inibição de enzimas envolvidas no metabolismo lipídico'. A apolipoproteína A-I (apo A-I) é o maior componente da partícula de lipoproteína de alta densidade (HDL), representando cerca de $45 \%$ de sua massa molecular ${ }^{2}$, e atua como cofator para a enzima lecitina colesterol acil transferase, e também como mediador na transferência do colesterol das células para as partículas de HDL, processos importantes para o transporte reverso do colesterol para o fígado². A apolipoproteína B (apo B) está presente nos quilomícrons como apo B-48 e nas lipoproteínas de densidade muito baixa (VLDL), lipoproteína de densidade intermediária (IDL), e lipoproteína de baixa densidade (LDL) como apo B-100, que é responsável pela ligação da lipoproteína ao seu receptor tecidual específico ${ }^{3}$. A apo B é a principal proteína funcional para o transporte de colesterol para as células periféricas ${ }^{4}$. Cerca de $90 \%$ da proteína na LDL se constitui de apo $\mathrm{B}^{5}$.

As partículas de LDL, IDL e VLDL apresentam uma molécula de apo B em sua estrutura ${ }^{3}$, e, dessa forma, a concentração plasmática de apo B indica o número total de partículas potencialmente aterogênicas, correlacionando-se com o nível de colesterol não-HDL ${ }^{6}$. A concentração plasmática de apo A-I é fortemente associada com HDLc, e a expressão de apo A-I pode ser responsável pela determinação dos níveis plasmáticos de HDLc 6 . Assim, o índice apo B/apo A-I representa o balanço entre as partículas de colesterol potencialmente aterogênicas ricas em apo $B$ e as partículas de colesterol antiaterogênicas ricas em apo A-I.

As pesquisas envolvendo o valor preditivo das apolipoproteínas A-I e B nas doenças ateroscleróticas surgiram há aproximadamente duas décadas, inicialmente

\section{Palavras-chave}

Apolipoproteínas B, apolipoproteínas A-I, prognóstico, fatores de risco.

Correspondência: Marinez Oliveira Sousa •

Av. Antônio Carlos, 6627 - UFMG - Faculdade de Farmácia - 31270-901

- Belo Horizonte, MG - Brasil

E-mail: marinez@farmacia.ufmg.br

Artigo recebido em 26/06/06; revisado recebido em 17/11/06; aceito em $17 / 11 / 06$. em estudos do tipo caso-controle ${ }^{7-9}$ e, posteriormente, em estudos prospectivos ${ }^{10-12}$. Atualmente, os níveis plasmáticos das apolipoproteínas A-I e B têm sido descritos como melhores preditores de doenças ateroscleróticas do que as concentrações de lípides e lipoproteínas ${ }^{6,13}$, e tem sido sugerido que o índice apo B/apo A-I representa um parâmetro superior para predição de risco cardiovascular do que outros índices lipídicos, como colesterol total/HDLc, LDLc/HDLc e colesterol não-HDL/HDLc ${ }^{14,15}$.

\section{Considerações analíticas}

As dosagens das apolipoproteínas apresentam algumas vantagens metodológicas quando comparadas com a quantificação de LDLc. Na maioria das vezes, o LDLc é quantificado usando-se a equação de Friedewald ${ }^{16}$, a qual fornece uma estimativa dos valores de LDLc, e depende das dosagens de colesterol total, triglicérides e HDLc ${ }^{17}$. Dessa forma, a estimativa pode incorporar os possíveis erros analíticos desses três parâmetros utilizados para o cálculo do $\mathrm{LDLC}^{6}$, aumentando assim a probabilidade de erros, com potencial impacto nas decisões clínicas ${ }^{18}$. Essa equação apresenta, ainda, várias limitações, e a estimativa do LDLc não pode ser ampliada para amostras apresentando níveis de triglicérides superiores a $400 \mathrm{mg} / \mathrm{dl}$, amostras contendo quilomícrons e para pacientes portadores de disbetalipoproteinemia ${ }^{19}$. Além disso, os estudos têm demonstrado que o método homogêneo para a dosagem de LDLc e a estimativa desses valores pela equação de Friedewald não apresentam resultados semelhantes $\mathrm{s}^{20-22}$.

As apolipoproteínas, por sua vez, podem ser medidas diretamente no plasma, por métodos exatos e precisos, padronizados internacionalmente ${ }^{23,24}$, utilizando-se material de referência comum para apo $A-I$ e apo $B$, o qual não está disponível para as medidas de HDLc e LDLc, e sem a interferência significativa de níveis elevados de triglicérides ${ }^{18}$. Os níveis plasmáticos das apolipoproteínas sofrem pouca influência de variáveis biológicas, enquanto os lípides apresentam flutuação dos níveis plasmáticos em resposta aos vários estímulos do controle metabólico ${ }^{4}$. Dessa forma, as variáveis pré-analíticas exercem menor influência nas dosagens das apolipoproteínas A-I e B, que podem ser quantificadas mesmo sem a necessidade de jejum prévio do paciente ${ }^{11,12,25}$.

No Brasil, os custos operacionais relacionados com as determinações das apolipoproteínas reduziram consideravelmente nos últimos anos, com a introdução no mercado brasileiro de conjuntos de reagentes nacionais com padronização internacional. Esse fato propiciou a realização das determinações das apolipoproteínas A-I e B por um maior número de laboratórios clínicos, por um custo mais 
acessível aos pacientes e com cobertura desses pela maioria dos convênios de saúde do país.

\section{Índice apo B/apo A-I e doença arterial coronariana (DAC)}

O valor preditivo das apolipoproteínas A-I e B para DAC está bem estabelecido e documentado na literatura. Níveis elevados de apo $B$, diminuição dos níveis de apo $A-I$ e aumento do índice apo B/apo A-I têm sido consistentemente associados ao risco de DAC $26-29$.

Quatro estudos prospectivos recentes destacam importantes evidências para a associação entre as apolipoproteínas A-I e B e a DAC. O Quebec Cardiovascular Study ${ }^{10}$ avaliou 2.155 homens canadenses e foi o primeiro estudo prospectivo a demonstrar que a apo B foi superior aos índices lipídicos convencionais na predição de risco cardiovascular. No mesmo estudo, após um acompanhamento de 13 anos $^{30}$, níveis plasmáticos elevados de apo B permaneceram como fator de risco independente para a predição de eventos coronarianos isquêmicos, e os autores concluíram que essa associação é maior em homens com níveis desejáveis de LDLc.

Na avaliação de cerca de 170 mil indivíduos suecos, o estudo AMORIS (Apolipoprotein-related Mortality Risk) ${ }^{11}$ demonstrou que a apo B se apresentou como melhor marcador de risco cardiovascular do que o LDLc, especialmente em indivíduos com níveis desejáveis de LDLc, independentemente do sexo. $O$ índice apo B/apo $A-I$ foi identificado nesse estudo como a variável isolada mais fortemente associada com o aumento do risco de infarto agudo do miocárdio (IAM) fatal, principalmente quando os níveis lipídicos estavam na faixa de valores desejáveis. Outros dados obtidos nesse estudo ${ }^{15}$ mostraram que o índice colesterol total/HDLc subestima consideravelmente o risco cardiovascular e que o índice apo B/apo A-I apresentou-se como a melhor variável relacionada a lípides para quantificar o risco coronariano, quando comparado com os índices colesterol total/HDLc, LDLc/HDLc e colesterol não-HDL/HDLc ${ }^{14}$.

Já o estudo INTERHEART ${ }^{31}$ avaliou cerca de $30 \mathrm{mil}$ indivíduos de 52 países e demonstrou que o índice apo B/apo A-I se mostrou mais fortemente associado com a predição de IAM do que vários fatores de risco convencionais, entre esses tabagismo, hipertensão, diabetes, estresse e obesidade abdominal, independentemente de sexo, idade e origem étnica. No estudo MONICA/KORA ${ }^{32}, 1.414$ homens e 1.436 mulheres sem história prévia de IAM foram avaliados durante 13 anos. O principal resultado desse estudo foi a observação da forte associação entre níveis aumentados de apo $B$ e risco aumentado para IAM, e a elevação dos níveis de apo A-I não foi significativamente associada ao baixo risco de IAM. Entretanto, a análise multivariada demonstrou que o índice apo B/apo A-I se associou fortemente ao risco de IAM, mesmo após ajustes para idade, índice de massa corporal, tabagismo, diabetes e hipertensão.

Contrariamente, o Women's Health Study ${ }^{33}$, onde cerca de 15 mil mulheres acima de 45 anos foram avaliadas por um período de 10 anos, observou que o colesterol não-HDL e o índice colesterol total/HDLc foram tão eficientes quanto as apolipoproteínas A-I e B e o índice apo B/apo A-I para predição do risco cardiovascular. No entanto, a apo B se comportou como o melhor parâmetro isolado para predição de futuros eventos cardiovasculares em mulheres.

Enquanto alguns estudos demonstraram a utilidade de níveis elevados de apo $\mathrm{B}$ como preditores de risco cardíaco ${ }^{32,34}$, outros atribuíram esse risco à diminuição dos níveis de apo $\mathrm{A}-\mathrm{I}^{35}$. Entretanto, prevalece um consenso na literatura de que o balanço entre as partículas aterogênicas e antiaterogênicas, refletido pelo índice apo B/apo A-I, representa um parâmetro adicional e importante para predição do risco cardiovascular, sendo atualmente considerado superior aos lípides, lipoproteínas e índices lipídicos convencionais ${ }^{14,36}$.

\section{Índice apo B/apo A-I e doença arterial em outros sítios anatômicos}

Ao contrário da vasta literatura disponível para o risco de DAC, a associação entre aterosclerose periférica com o índice apo B/apo A-I ainda não está bem consolidada, sendo essa correlação descrita em poucos estudos. Em 1984, McConathy e cols. ${ }^{37}$ demonstraram que as apolipoproteínas A-I e B foram importantes para diferenciar indivíduos com doença arterial obstrutiva periférica (DAOP) de indivíduos hígidos em um grupo de mulheres, quando os dados foram analisados em conjunto com as dosagens de colesterol total e triglicérides. Por sua vez, no estudo prospectivo de Schmidt e cols. ${ }^{38}$, envolvendo 391 homens adultos, acompanhados durante 6,6 anos, observou-se que o índice apo B/apo A-I apresentou associação com aterosclerose na artéria femoral e com risco aumentado para doenças cardiovasculares, comportando-se como um marcador de risco superior ao LDLc. Dois estudos de coorte transversal conduzidos em nosso laboratório (dados não-publicados) mostraram resultados controversos em relação ao índice apo B/apo A-I e aterosclerose periférica. No primeiro estudo, o índice apo B/apo A-I se apresentou significativamente elevado em pacientes jovens com aterosclerose periférica em diferentes sítios anatômicos (membros superiores, inferiores e retina), quando comparados com indivíduos hígidos. Contrariamente, outro estudo avaliando pacientes idosos apresentando doença arterial periférica apenas em membros inferiores, o índice apo B/apo A-I não apresentou contribuição adicional quando os pacientes foram comparados com indivíduos hígidos. Apesar de as diferenças na composição dos grupos terem sido significativas (idade, fatores de risco, sítios anatômicos comprometidos etc), esses dados reforçam a idéia da necessidade de estudos adicionais envolvendo o índice apo B/apo A-I e doenças ateroscleróticas periféricas.

Com relação à aterosclerose em artérias cerebrais, as evidências são recentes e escassas. No estudo de Bhatia e cols. $^{39}$, envolvendo 261 pacientes com isquemia transitória prévia, acompanhados por 10 anos, o índice apo B/apo A-I apresentou-se como o melhor preditor independente para AVC isquêmico nesse grupo de pacientes, seguido pela apo $B$, quando os dados foram analisados em conjunto com lípides, lipoproteínas e índices lipídicos tradicionais. Essa observação foi confirmada pelo estudo AMORIS ${ }^{40}$, o qual demonstrou uma forte associação entre o índice apo B/apo A-I e o risco de desenvolvimento de acidente vascular cerebral 


\section{Atualização Clínica}

(AVC), isquêmico ou não, sugerindo que essa associação seria semelhante à observada para DAC. A análise multivariada deste último estudo também demonstrou que o índice apo B/apo A-I foi melhor preditor de risco do que os índices convencionais colesterol total/HDLc e LDLc/HDLc, constituindo-se em um melhor marcador de eventos isquêmicos.

\section{Índice apo B/apo A-I e agentes hipolipemiantes}

Estudos envolvendo o índice apo B/apo A-I têm demonstrado que os agentes hipolipemiantes, sobretudo as estatinas, exercem efeitos relevantes no perfil apolipoprotéico, algumas com significativa redução nos níveis de apo $B^{41}$, outras com aumento nos níveis de apo A-I e outras ainda atuando nas duas apolipoproteínas ${ }^{6,42,43}$. Dessa forma, o tratamento com esses agentes pode apresentar um grande potencial em corrigir o balanço anormal entre lipoproteínas aterogênicas e antiaterogênicas que está intimamente associado com o risco cardiovascular.

O uso das apolipoproteínas A-I e B, assim como o índice apo B/apo A-I, como meta terapêutica para agentes hipolipemiantes, no entanto, ainda não está completamente consolidado pela literatura. A inclusão desses parâmetros nos consensos americano e europeu para prevenção de doenças cardiovasculares tem sido alvo de controvérsia e discussão entre os pesquisadores da área ${ }^{44}$. 0 maior problema destacado seria o fato de que, uma vez definida a meta terapêutica para o índice apo B/apo A-I, seria melhor diminuir os valores do numerador (apo B) ou aumentar os valores do denominador (apo A-I)? As evidências a favor da diminuição dos níveis de apo B são muito fortes ${ }^{45,46}$, mas o aumento dos níveis de apo A-I também é importante para diminuir o risco cardíaco ${ }^{28}$. O mérito dos benefícios dos agentes hipolipemiantes no perfil apolipoprotéico não está sendo questionado [ver ref. 44], mas um bom marcador de risco cardiovascular não necessariamente precisa indicar uma meta para terapia. Dessa forma, a elevação do índice apo B/apo A-I pode perfeitamente ser usada apenas como um marcador de risco aumentado. e não necessariamente como alvo para terapia com agentes hipolipemiantes.

\section{Pontos de corte}

Em 2004, Walldius e cols. ${ }^{6}$ sugeriram pontos de corte para o índice apo B/apo A-I de 0,9 e 0,8 para homens e mulheres, respectivamente, demonstrando que valores superiores a esses representariam risco aumentado para doenças cardiovasculares. Esses valores têm sido confirmados por outras pesquisas $37,47,48$ e os resultados dos estudos AMORIS ${ }^{5}$ e INTERHEART ${ }^{31}$ estabeleceram faixas de risco para infarto agudo do miocárdio, mostrados na tabela 1.

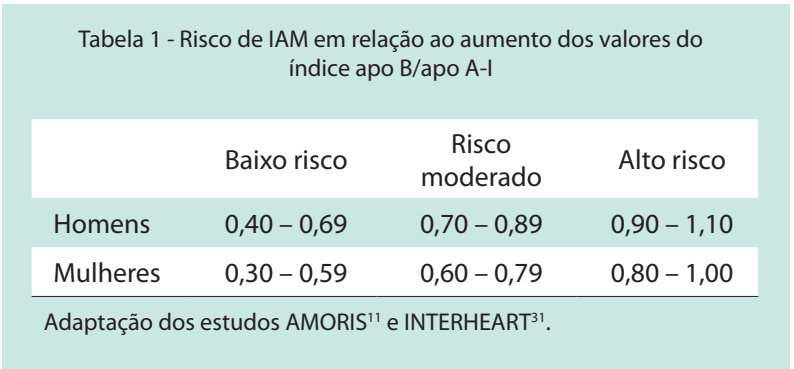

\section{Conclusão}

Com base nas recentes evidências das vantagens do uso das apolipoproteínas A-I e B como marcadores de risco cardiovascular, o índice apo B/apo A-I emerge como importante parâmetro complementar para avaliação desse risco, especialmente em indivíduos normolipêmicos, com potencial importância para aplicação na monitoração de pacientes de alto risco em terapia com agentes hipolipemiantes no futuro.

Financiamento: Capes e CNPq.

Potencial Conflito de Interesses

Declaro não haver conflitos de interesses pertinentes.

\section{Referências}

1. Beisiegel U. Lipoprotein metabolism. Eur Heart J. 1998; 19 (Suppl A): S2023.

2. Frank PG, Marcel YL. Apolipoprotein A-I: structure-function relationships.J Lipid Res. 2000; 41:853-72.

3. Packard CJ, Shepherd J. Lipoprotein heterogeneity and apolipoprotein B metabolism. Arterioscler Thromb Vasc Biol. 1997; 17: 3542-56.

4. Rifai N, Bachorik PS, Alberts JJ. Lipids, lipoproteins, and apolipoproteins. In Burtis CA, Ashwood ER (eds). Tietz - textbook of clinical chemistry. 3rd ed. Philadelphia: Saunders; 1999. p. 809-61.

5. Walldius G, Jungner I. The apoB/apoA-I ratio: a strong, new risk factor for cardiovascular disease and a target for lipid-lowering therapy - a review of the evidence. J Intern Med. 2006; 259:493-519.

6. Walldius G, Jungner I. Apolipoprotein B and apolipoprotein A-I: risk indicators of coronary heart disease and targets for lipid-modifying therapy. JIntern Med.
$2004 ; 255: 188-205$

7. Avogaro P, Bittolo BG, Cazzolato G, Quinci GB. Are apolipoproteins better discriminators than lipids for atherosclerosis? Lancet. 1979; 1: 901-3.

8. Sniderman AD, Wolfson C, Teng B, Franklin FA, Bachorik OS, Kwiterovich $P O \mathrm{Jr}$. Association of hyperapobetalipoproteinemia with endogenous hypertriglyceridaemia and atherosclerosis. Ann Intern Med. 1982; 97: 833-9.

9. Durrington PN, Hunt L, Ishola M, Kane J, Stephens WP. Serum apolipoproteins $\mathrm{Al}$ and $\mathrm{B}$ and lipoproteins in middle-aged men with and without previous myocardial infarction. Br Heart J. 1986; 56: 206-12.

10. Lamarche B, Moorjani S, Lupien PJ, Cantin B, Bernard PM, Dagenais GR, et al. Apolipoprotein Al and B levels and the risk of ischaemic heart disease during a five-years follow-up of men in the Quebec cardiovascular study. Circulation. 1996; $94: 273-8$. 
11. Walldius G, Jungner I, Holme I, Aastveit AH, Kolar W, Steiner E. High apolipoprotein B, low apolipoprotein A-I, and improvement in the prediction of fatal myocardial infarction (AMORIS Study): a prospective study. Lancet. 2001; 358:2026-33.

12. Talmud PJ, Hawe E, Miller GJ, Humphries SE. Non-fasting apolipoprotein B and triglyceride levels as a useful predictor of coronary heart disease risk in middle-aged UK men. Arterioscler Thromb Vasc Biol. 2002; 22: 1918-23.

13. van Lennep JE, Westerveld HT, van Lennep HW, Zwinderman AH, Erkelens $\mathrm{DW}$, van der Wall EE. Apolipoprotein concentrations during treatment and recurrent coronary artery disease events. Arterioscler Thromb Vasc Biol. 2000; $20: 2408-13$

14. Walldius G, Jungner I, Aastveit AH, Holme I, Furberg CD, Sniderman AD. The apoB/apoA-I ratio is better than cholesterol ratios to estimate the balance between plasma proatherogenic and antiatherogenic lipoproteins and to predict coronary risk. Clin Chem Lab Med. 2004; 42: 1355-63.

15. Sniderman AD, Jungner I, Holme I, Aastveit A, Walldius G. Errors that result from the TC/HDLC ratio rather than the apoB/apoA-I ratio to identify the lipoprotein-related risk of vascular disease. J Intern Med. 2006; 259: 455-61.

16. Friedewald WT, Levy Rl, Fredrickison DS. Estimation of the concentration of low density lipoprotein cholesterol in plasma, without the use of preparative ultracentrifugue. Clin Chem. 1972; 18:499-552.

17. National Cholesterol Education Program Recommendations on lipoprotein Measurement. From the Working Group on Lipoprotein Measurement. NIH Publication no 95-3044. Bethesda, MD: National Heart, Lung, and Blood Institute, 1995.

18. Marcovina S, Packard J. Measurement and meaning of apolipoprotein Al and apolipoprotein B plasma levels. J Intern Med. 2006; 259:437-46.

19. McNamara JR, Conh JS, Wilson PWF. Calculated values of low-density lipoprotein in the assessment of lipid abnormalities and coronary disease risk. Clin Chem. 1990; 36: 36-42.

20. Esteban-Salán M, Guimón-Berdesi A, de la Viuda-Unzueta JM. Analytical and clinical evaluation of two homogeneous assays for LDL-cholesterol in hyperlipidemic patients. Clin Chem. 2000; 46: 1121-31.

21. Yu HH, Ginsbrug GS, Harris N. Evaluation and clinical application of a direct low density lipoprotein cholesterol assay in normolipemic and hyperlipidemic adults. Am J Cardiol. 1997; 80:1295-9.

22. Cordova CMM, Schneider CR, Juttel ID, Cordova MM. Avaliação da dosagem direta do colesterol-LDL em amostras de sangue de 10.664 pacientes em comparação com o uso da fórmula de Friedewald. Arq Bras Cardiol. 2004; 83:476-81.

23. Marcovina SM, Alberts JJ, Henderson LO, Hannon WH. International Federation of Clinical Chemistry standardization project for measurements of apolipoproteins A-I and B. III comparability of apolipoprotein A-I values by use of international reference material. Clin Chem. 1993; 39:773-81.

24. Marcovina SM, Alberts JJ, Kennedy H, Mei JV, Henderson LO, Hannon WH. International Federation of Clinical Chemistry standardization project for measurements of apolipoproteins A-I and B. IV comparability of apolipoprotein B values by use of international reference material. Clin Chem. 1994; 40: 586-92.

25. Durington PN. Can measurement of apolipoprotein B replace the lipid profile in the follow-up of patients with lipoprotein disorders? Clin Chem. 2002; 48 : 401-2.

26. Bolibar I, Von Eckardstein A, Assmann G, Thompson S, ECAT Angina Pectoris Study Group - European Concerted Action on Thrombosis and Disabilities. Short-term prognostic value of lipid measurements in patients with angina pectoris. The ECAT Angina Pectoris Study Group: European Concerted Action on Thrombosis and Disabilities. Thromb Haemost. 2000; 84: 955-60.

27. Walldius $G$, Jungner I. Apolipoproteins are new and better risk indicators of myocardial infarction. Lakartidningen. 2004; 101:1188-94.

28. Chan DC, Watts GF. Apolipoproteins as markers and managers of coronary risk. QJM. 2006; 99: 277-87.

29. Zambon A, Brown BG, Deeb SS, Brunzell JD. Genetics of apolipoprotein B and apolipoprotein Al and premature coronary artery disease. J Intern Med. 2006; 259: 473-80.
30. St-Pierre AC, Cantin B, Dagenais GR, Mauriege P, Bernard PM, Despres $J P$, et al. Low-density lipoprotein subfractions and the long-term risk of ischemic heart disease in men: 13-year follow-up data from the Québec Cardiovascular Study. Arterioscler Thromb Vasc Biol. 2005; 25: 553-9.

31. Yusuf S, Hawken S, Öunpuu S, Dans T, Avezum A, Lanas F, et al. Effect of potentially modifiable risk factors associated with myocardial infarction in 52 countries (the INTERHEART study): case-control study. Lancet. 2004; 364 937-52.

32. Meisinger C, Loewel H, MrazW, Koenig W. Prognostic value of apolipoprotein $B$ and $A-l$ in the prediction of myocardial infarction in middle-aged men and women: results from the MONICA/KORA Augsburg cohort study. Eur Heart J. $2005 ; 26: 271-8$

33. Ridker PM, Rifai N, Cook NR, Bradwin G, Buring JE. Non-HDL cholesterol, apolipoproteins A-I and B100, standard lipid measures, lipid ratios, and CRP as risk factors for cardiovascular disease in women. JAMA. 2005;294:326-33.

34. Westerveld HT, Roeters Van Lennep JE, Roeters Van Lennep HWO, Liem AH de Boo JA, van der Schown YT, et al. Apolipoprotein B and coronary artery disease in women: a cross-sectional study in women undergoing their first coronary angiography. Arterioscler Thromb Vasc Biol. 1998; 18:1101-7.

35. Luc G, Bard JM, Ferrières J, Evans A, Amouyel P, Arvelier D, et al. Value of HDL cholesterol, apolipoprotein A-I, lipoprotein A-I, and lipoprotein A-I/A-II in prediction of coronary heart disease. The PRIME Study. Arterioscler Thromb Vasc Biol. 2002; 22: 1155-61.

36. Kim HK, Chang SA, Choi EK, Kim YJ, Kim HS, Sohn DW, et al. Association between plasma lipids, and apolipoproteins and coronary artery disease: a cross-sectional study in a low-risk Korean population. Int J Cardiol. 2005; 101:435-40.

37. McConathy WJ, Greenhalgh RM, Alaupovic P, Woolcock NE, Laing SP, Lund $V$, et al. Plasma lipid and apolipoprotein profiles of women with two types of peripheral arterial disease. Atherosclerosis. 1984; 50:295-306.

38. Schmidt C, Fagerberg B, Wikstrand J, Hulthe J. ApoB/apoA-I ratio is related to femoral artery plaques and is predictive for future cardiovascular events in healthy men. Atherosclerosis. 2006; 189 (1): 178-85.

39. Bhatia M, Howard SC, Clark TG, Neale R, Qizilbash N, Murphy MF, et al. Apolipoproteins as predictors of ischaemic stroke in patients with a previous transient ischaemic attack. Cerebrovasc Dis. 2006; 21:323-8.

40. Walldius $G$, Aastveit $A H$, Jungner I. Stroke mortality and the apoB/apoA-I ratio: results of the AMORIS prospective study. J Intern Med. 2006; 259: 259-66.

41. Ballantyne CM, Andrews TC, Hsia JA, Kramer JH, Shear C, ACCESS study Group Atorvastatin Comparative cholesterol Efficacy and Safety Study. Correlation of non-high-density lipoprotein cholesterol with apolipoprotein B: effect of 5 hydroxymethylglutaryl coenzime A reductase inhibitors on nonhigh- density lipoprotein cholesterol levels. Am J Cardiol. 2001; 88: 265-9.

42. Endreas M. Statins and stroke. J Cereb Blood Flow Metab. 2005; 25: 1093 110.

43. Charlton-Menys V, Durrington P. Apolipoproteins AI and B as therapeutic targets. J Intern Med. 2006; 259: 462-72.

44. Faergeman O. Apolipoproteins and guidelines for prevention of cardiovascular disease. J Intern Med. 2006; 259:434-6.

45. Baigent C, Keech A, Kearney PM, Blackwell L, Buck G, Pollicino C, et al. Efficacy and safety of cholesterol lowering treatment: prospective metaanalysis of data from 90.056 participants in 14 randomised trials of statins. Lancet. 2005; 366: 1267-78.

46. Sniderman AD, Furberg CD, Keech A, Roeters van Lennep JE, Frohlich J Jungner I. Apolipoproteins versus lipids as indices of coronary risk and as targets for statin treatment. Lancet. 2003;361:777-80.

47. WallenfeldtK, Bokemark L, Wikstrand J, Hulthe J, Fagerberg B. Apolipoprotein $B / A p o l i p o p r o t e i n A-I$ relation to the metabolic syndrome and change in carotid artery intima-media thickness during 3 years in middle-aged men. Stroke. 2004; 35: 2248-52.

48. Thompson A, Danesh J. Associations between apolipoprotein $B$ apolipoprotein $\mathrm{Al}$, the apolipoprotein $\mathrm{B} / \mathrm{Al}$ ratio and coronary artery disease: a literature-based meta-analysis of prospective studies. J Intern Med. 2006 259: 481-92. 